\title{
Triviality of quantum electrodynamics revisited
}

\author{
D. Djukanovic, ${ }^{1}$ J. Gegelia, ${ }^{2,3}$ and Ulf-G. Meißner ${ }^{4,2}$ \\ ${ }^{1}$ Helmholtz Institute Mainz, University of Mainz, D-55099 Mainz, Germany \\ ${ }^{2}$ Institute for Advanced Simulation, Institut für Kernphysik and Jülich Center for Hadron Physics, \\ Forschungszentrum Jülich, D-52425 Jülich, Germany \\ ${ }^{3}$ Tbilisi State University, 0186 Tbilisi, Georgia \\ ${ }^{4}$ Helmholtz Institut für Strahlen- und Kernphysik and Bethe Center \\ for Theoretical Physics, Universität Bonn, D-53115 Bonn, Germany
}

(Dated: 31 May, 2017)

\begin{abstract}
Quantum electrodynamics is considered to be a trivial theory. This is based on a number of evidences, both numerical and analytical. One of the strong indications for triviality of QED is the existence of the Landau pole for the running coupling. We show that by treating QED as the leading order approximation of an effective field theory and including the next-to-leading order corrections, the Landau pole is removed. Therefore, we conclude that the conjecture, that for reasons of selfconsistency, QED needs to be trivial is a mere artefact of the leading order approximation to the corresponding effective field theory.
\end{abstract}

PACS numbers: 03.70.+k, 11.10.Gh, 14.70.Bh

The concept of triviality in quantum field theories originates from papers by Landau and collaborators studying the asymptotic behaviour of the photon propagator in quantum electrodynamics (QED) [1, 2] (for a review see e.g. Ref. [3]). Resumming the leading logarithmic contributions they found that the photon propagator has a pole at large momentum transfer. If this pole persists also in non-perturbative calculations then to avoid the apparent inconsistency QED has to be a non-interacting, i.e. trivial, theory. In calculations applying a finite cutoff this problem manifests itself as a singularity in the bare coupling for a finite value of the cutoff. It is therefore impossible to remove the cutoff unless the renormalized coupling vanishes.

The standard model, in a modern point of view, is a leading order approximation to an effective field theory (EFT) 4]. While the effective Lagrangian contains an infinite number of local interactions consistent with the underlying symmetries, at low energies the contributions in physical quantities of the interactions with coupling constants of negative mass dimensions (i.e. nonrenormalizable interactions in the traditional sense) are suppressed by powers of the energy divided by a large scale characterising those degrees of freedom which are not explicitly taken into account in the effective theory. In the framework of EFT the solution of the leading order Wilson renormalization group equations might be obstructed at very large cutoffs, however, this should not be a hard problem because of irrelevant interactions or omitted fields being important at short distances [4]. Therefore inconsistencies in the renormalization group analysis of renormalizable quantum field theories, like QED or $\phi^{4}$ theory of self-interacting scalars, might be absent in the corresponding EFT framework.

In this letter we address the consequences of treating QED as a leading order approximation of an EFT for the problem of triviality. To that end we analyse the contributions of the next-to-leading order interaction, i.e. dimension five operator, the well-known Pauli term. We start with the most general U(1) locally gauge invariant effective Lagrangian of the electron (and the positron) field $\psi$ interacting with the electromagnetic field $A_{\mu}$

$$
\begin{aligned}
\mathcal{L} & =-\frac{1}{4} F^{\mu \nu} F_{\mu \nu}+\bar{\psi}(i \not D-m) \psi \\
& +\frac{i \kappa}{2} \bar{\psi}\left(\gamma^{\mu} \gamma^{\nu}-\gamma^{\nu} \gamma^{\mu}\right) \psi F_{\mu \nu}+\mathcal{L}_{\mathrm{ho}}
\end{aligned}
$$

where $m$ is the (bare) electron mass, $e$ is the (bare) electromagnetic charge, $F_{\mu \nu}=\partial_{\mu} A_{\nu}-\partial_{\nu} A_{\mu}, D_{\mu}=\partial_{\mu}-i e A_{\mu}$ and $\mathcal{L}_{\text {ho }}$ contains an infinite number of terms with operators of order six and higher. We assume that the contributions of these terms in the photon self-energy are suppressed compared to those of the Pauli term. We remark here that the standard QED Lagrangian given by the first line of Eq. (11) describes the experimental data very well. From the modern point of view this is because the contributions of terms in the second line are beyond the current accuracy of the data. In particular, the anomalous magnetic moment of the electron in standard QED gets contributions only from loop diagrams and its calculated value agrees with the experiment very well suggesting that the Pauli term is suppressed by a scale larger than $4 \times 10^{7} \mathrm{GeV}$ [4].

The scale-dependent renormalized running coupling $e_{R}\left(q^{2}\right)$ can be defined by the following relation 1

$$
D^{\mu \nu}(q) e^{2}=-\frac{1}{q^{2}}\left(g^{\mu \nu}-\frac{q^{\mu} q^{\nu}}{q^{2}}\right) e_{R}^{2}\left(q^{2}\right),
$$

\footnotetext{
${ }^{1}$ We carried out all calculations in Landau gauge, however, the results are gauge independent.
} 


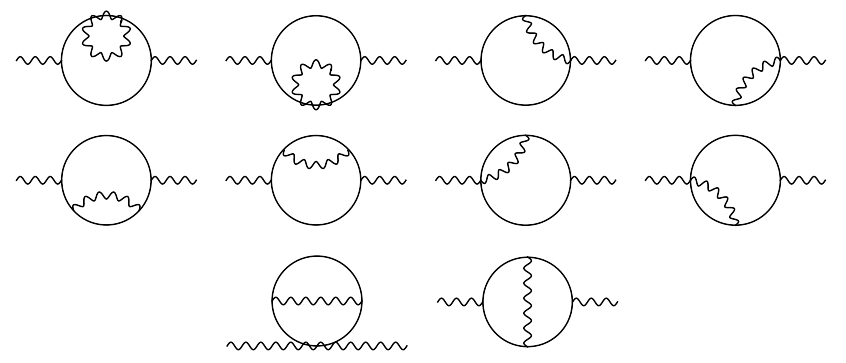

FIG. 1. Two-loop diagrams contributing in the photon selfenergy. The solid and wiggly lines correspond to the electron and photon propagators, respectively.
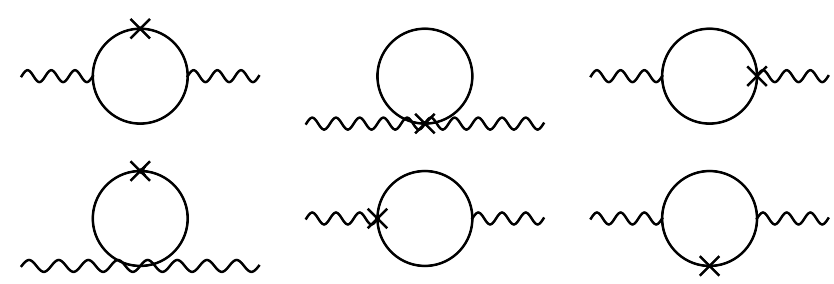

FIG. 2. One loop counter term diagrams contributing in the photon self-energy at two-loop order. The solid and wiggly lines correspond to the electron and photon propagators, respectively. The crosses denote the counter terms of the oneloop order. Diagrams with ghost propagators are not shown.

where $D^{\mu \nu}(q)$ is the dressed propagator of the bare photon field. Calculating the dressed propagator at one-loop order we obtain for $-q^{2} \gg m^{2}$ :

$$
e_{R}^{2}\left(q^{2}\right)=\frac{e_{r}^{2}}{1-\frac{e_{r}^{2}+2 \kappa^{2} q^{2}}{12 \pi^{2}} \ln \frac{-q^{2}}{m^{2}}+c_{R} q^{2}} .
$$

Here, $e_{r}$ is the renormalized coupling at $q^{2}=-m^{2}$ for $c_{R}=0$, where $c_{R}$ is a renormalized coupling constant of the higher-order Lagrangian $\mathcal{L}_{\text {ho }}$. It is suppressed by two orders of some large scale. For $\kappa=c_{R}=0$ the running coupling has the well-known pole singularity at (the Landau pole)

$$
q_{L}^{2}=-m^{2} \exp \left[12 \pi^{2} / e_{r}^{2}\right]
$$

While this pole appears at extremely high energies, reducing its practical importance to nil, it is still a problem if present in the full theory [3]. For reasonable values of $\kappa \gg 1 / \sqrt{-q_{L}^{2}}$ the Landau pole is absent remedying the inconsistency at the level of an EFT.
In renormalizable theories only logarithmic divergences contribute to the renormalization of coupling constants and therefore there is a direct correspondence between the Gell-Mann-Low [5] and the Wilsonian renormalization group approaches [6]. As a result, the presence of the Landau pole in the expression of the running renormalized coupling authomatically leads to the pole in the bare coupling as a function of the cutoff parameter. However, in an EFT with non-renormalizable interactions the direct link between the two renormalization group equations is lost and therefore the Wilsonian renormalization group approach requires additional study of the cutoff dependence. We investigate the cutoff dependence of the bare electromagnetic coupling in our model by applying the higher derivative regularization $[7,[8]$ which preserves the local U(1) gauge invariance. Notice that dimensional regularization is not suitable here as it discards the power-law divergences. In addition to the fields in conventional QED, we introduce scalar (i.e. commuting) ghost fields $\bar{\xi}$ and $\xi$ which regulate the one-loop counter term diagrams contributing to the photon self-energy at two-loop order. The effective Lagrangian generating one and two-loop diagrams, contributing to our calculation of the photon self-energy up to two-loop $e^{2} \kappa^{2}$ order, which are all finite for finite $\Lambda$, is given by:

$$
\begin{aligned}
\mathcal{L}_{\mathrm{HDR}} & =-\frac{1}{4} F^{\mu \nu}\left(1+\frac{\partial^{2}}{\Lambda^{2}}\right)^{2} F_{\mu \nu} \\
& +\frac{1}{2} \bar{\psi}(i \not D-m)\left(1+\frac{D^{2}}{\Lambda^{2}}\right)^{3} \psi+\text { h.c. } \\
& +\frac{1}{2} \bar{\xi}(i \not D-m)\left(1+\frac{D^{2}}{\Lambda^{2}}\right)^{3} \xi+\text { h.c. } \\
& +\frac{i \kappa}{2} \bar{\psi}\left(\gamma^{\mu} \gamma^{\nu}-\gamma^{\nu} \gamma^{\mu}\right) \psi F_{\mu \nu}+\mathcal{L}_{\mathrm{ho}} .
\end{aligned}
$$

The bare electromagnetic coupling as a function of the cutoff satisfies a renormalization group equation which up to the level of accuracy of our calculation has the form:

$\frac{d \alpha(\Lambda)}{d \ln \Lambda}=A_{1} \alpha^{2}(\Lambda)+\kappa^{2} \alpha^{2}(\Lambda) \Lambda^{2}\left(2 A_{2}+A_{3}+2 A_{3} \ln \Lambda / m\right)$,

where $\alpha(\Lambda)=e^{2} /(4 \pi)$, the coefficient $A_{1}$ is given by oneloop diagrams and $A_{2}$ and $A_{3}$ are extracted from twoloop calculations. Notice that there are no power-law divergences at one-loop order and all terms suppressed by powers of $m / \Lambda$ have been dropped in our calculations as they are negligible for large values of $\Lambda$.

The solution to Eq. (6) is given by

$$
\alpha(\Lambda)=\frac{\alpha_{0}}{1-\alpha_{0} \ln \frac{\Lambda}{m}\left(A_{1}+A_{3} \kappa^{2} \Lambda^{2}\right)+\alpha_{0} \ln \frac{\Lambda_{0}}{m}\left(A_{1}+A_{3} \kappa^{2} \Lambda_{0}^{2}\right)-\alpha_{0} A_{2} \kappa^{2}\left(\Lambda^{2}-\Lambda_{0}^{2}\right)},
$$


where $\alpha_{0}=\alpha\left(\Lambda_{0}\right)$ is the bare coupling at some fixed cutoff $m<\Lambda_{0}<\Lambda$.

For $\Lambda \gg \Lambda_{0}$ we have

$$
\alpha(\Lambda)=\frac{\alpha_{0}}{1-\alpha_{0} \ln \frac{\Lambda}{m}\left(A_{1}+A_{3} \kappa^{2} \Lambda^{2}\right)-\alpha_{0} A_{2} \kappa^{2} \Lambda^{2}} .
$$

For $\kappa=0$ and positive $A_{1}$ the expression in Eq. (8) has a pole at

$$
\Lambda_{\mathrm{P}}=m \exp \left[\frac{1}{A_{1} \alpha_{0}}\right] .
$$

This pole, if remaining in the non-perturbative full expressions of the bare coupling, prevents the $\Lambda \rightarrow \infty$ limit unless $\alpha_{0} \equiv 0$, thus leaving us with a non-interacting theory. In renormalizable theories where only logarithmic divergences contribute in the renormalization of coupling constants, there is a close correspondence between the Gell-Mann-Low and the Wilsonian renormalization group approaches manifesting itself in a direct relation of Eqs. (4) and (8) valid for standard QED.

Using FeynCalc [9, 10] and Form [11] and applying the method of dimensional counting of Ref. [12] we have calculated the logarithmically divergent contributions to the photon self-energy generated by one-loop diagrams, and the quadratic divergences generated by the two-loop diagrams, shown in Fig. $1^{2}$ and by the corresponding counter term diagrams, shown in Fig. 2, Our results $\operatorname{read} \sqrt[3]{3}$

$$
\begin{aligned}
A_{1} & =\frac{2}{3 \pi} \simeq 0.212, \quad A_{3}=-\frac{7}{40 \pi^{3}} \simeq-0.0056, \\
A_{2} & =-\frac{5491889}{201600 \pi^{3}}-\frac{2194315}{104976 \pi}+\frac{8593 \ln 3}{5832 \sqrt{3} \pi^{2}} \\
& +\frac{1508572 \psi^{(1)}\left(\frac{1}{3}\right)-303413 \psi^{(1)}\left(\frac{1}{6}\right)}{17496 \pi^{3}} \\
& +\frac{8593 i}{972 \sqrt{3} \pi^{3}}\left[2 \operatorname{Li}_{2}\left(-\frac{i}{\sqrt{3}}\right)-2 \operatorname{Li}_{2}\left(\frac{i}{\sqrt{3}}\right)\right. \\
& \left.-\operatorname{Li}_{2}\left(\frac{1}{2}-\frac{i}{2 \sqrt{3}}\right)+\operatorname{Li}_{2}\left(\frac{1}{2}+\frac{i}{2 \sqrt{3}}\right)\right] \simeq 0.0040,
\end{aligned}
$$

where $\mathrm{Li}_{2}$ and $\psi^{(1)}$ are the dilogarithm and trigamma functions, respectively. For the above values of $A_{1}, A_{2}$ and $A_{3}$, and for natural values of $\kappa \gg 1 / \Lambda_{\mathrm{P}}$, in the denominator of Eq. (8) the $A_{3}$ term is larger than the $A_{1}$ and $A_{2}$ terms and the negative sign of $A_{3}$ guarantees that $\alpha(\Lambda)$ has no pole.

To conclude, we have shown that the problem of triviality in QED can be attributed to QED being a leading order approximation of an effective field theory. We have shown that already at next-to-leading order, i.e. adding the Pauli term to QED, the Landau pole disappears thereby obviating the need for QED to be a trivial theory.

This work was supported in part by DFG and NSFC through funds provided to the Sino-German CRC 110 "Symmetries and the Emergence of Structure in QCD" (NSFC Grant No. 11621131001, DFG Grant No. TRR110), by the Georgian Shota Rustaveli National Science Foundation (grant FR/417/6-100/14) and by the CAS President's International Fellowship Initiative (PIFI) (Grant No. 2017VMA0025).

[1] L. D. Landau, A. A. Abrikosov and J. M. Khalatnikov, Dokl. Akad. Nauk SSSR 95(1954) 773; 95(1954)1177; 96 (1954) 261.

[2] L. D. Landau, in: Niels Bohr and the Development of Physics, ed. W. Pauli (Pergamon, London, 1955); and works cited in there.

[3] D. J. E. Callaway, Phys. Rept. 167, 241 (1988).

[4] S. Weinberg, "The Quantum Theory Of Fields. Vol. 1,2." Cambridge, UK: Univ. Pr. (1995).

[5] M. Gell-Mann and F. E. Low, Phys. Rev. 95, 1300 (1954).

[6] K. G. Wilson and J. B. Kogut, Phys. Rept. 12, 75 (1974).

[7] L. D. Faddeev and A. A. Slavnov, Front. Phys. 50, 1 (1980) [Front. Phys. , 1 (1991)].

[8] D. Djukanovic, M. R. Schindler, J. Gegelia and S. Scherer, Phys. Rev. D 72, 045002 (2005).

[9] R. Mertig, M. Bohm and A. Denner, Comput. Phys. Commun. 64, 345 (1991).

[10] V. Shtabovenko, R. Mertig and F. Orellana, Comput. Phys. Commun. 207, 432 (2016).

[11] J. A. M. Vermaseren, math-ph/0010025

[12] J. Gegelia, G. S. Japaridze and K. S. Turashvili, Theor. Math. Phys. 101, 1313 (1994) [Teor. Mat. Fiz. 101, 225 (1994)].

[13] T. Huber and D. Maitre, Comput. Phys. Commun. 178, 755 (2008).

\footnotetext{
2 Notice here that due to the higher derivative regularization there are interaction vertices not present in standard QED.
} 